\title{
COMPARATIVE STUDY OF MEDIUM DAMPED AND DETUNED LINEAR ACCELERATOR STRUCTURES*
}

\author{
J.-F. Ostiguy, K.-Y. Ng, FNAL, Batavia, IL 60510, USA \\ Z. Li, R. Jones, SLAC, Stanford, CA 94309, USA, N. Kroll, UCSD
}

\begin{abstract}
Long range wakefields are a serious concern for a future linear collider based on room temperature accelerating structures. They can be suppressed either by detuning and or local damping or with some combination of both strategies. Detuning relies on precisely phasing the contributions of the dipole modes excited by the passage of a single bunch. This is accomplished by controlling individual mode frequencies, a process which dictates individual cell dimensional tolerances. Each mode must be excited with the correct strength; this in turn, determines cell-tocell alignment tolerances. In contrast, in a locally damped structure, the modes are attenuated at the cell level. Clearly, mode frequencies and relative excitation become less critical in that context; mechanical fabrication tolerances can be relaxed. While local damping is ideal from the standpoint of long range wakefield suppression, this comes at the cost of reducing the shunt impedance and possibly unacceptable localized heating. Recently, the Medium Damped Structure (MDS), a compromise between detuning and local damping, has generated some interest. In this paper, we compare a hypothetical MDS to the NLC Rounded Damped Detuned Structure (RDDS) and investigate possible advantages from the standpoint fabrication tolerances and their relation to beam stability and emittance preservation.
\end{abstract}

\section{INTRODUCTION}

In linear accelerating structures, wakefield strength (both short range and long range) depends on the structure transverse dimension and the bunch charge. To mitigate the short range wake, a standard strategy is to spread out the total charge by accelerating a bunch train comprised of $N$ equally spaced bunches rather than a single bunch. While this reduces short-range single bunch wakefield intensity by a factor $N$, it introduces the potential for beam breakup (BBU) due to bunch-to-bunch interactions via long range wakefields.

The NLC accelerating structures are of the traveling wave type, operating at $11.4 \mathrm{GHz}$ (X-band). The NLC design specifies a $400 \mathrm{~ns}$ rf pulse length to the accelerating structures and a bunch train is comprised of 1901 $\mathrm{nC}$ bunches separated from each other by $1.4 \mathrm{~ns}$. A point charge passing through a structure with a radial offset excites higher order, predominantly dipole, modes. The transverse deflection experienced by each bunch grows exponentially with its position within the train. To limit this

\footnotetext{
* Work supported by Universities Research Association, under contract DE-AC02-76CH03000 with the U. S. Department of Energy.
}

growth, it is necessary to reduce the intensity of the induced transverse wake, on a time scale shorter than the bunch-tobunch spacing. This can be accomplished using two different strategies. The first one, referred to as localized damping, consists in coupling the deflecting mode energy out of each cell via a small aperture and subsequently redirect it into a suitable absorber (usually $\mathrm{SiC}$ ). Unfortunately, it is difficult to couple out significant amount of higher order mode energy without reducing overall acceleration efficiency. In terms of cell modes, mode conversion from the fundamental accelerating mode to very high order modes due to the perturbation created by the presence of the opening results in localized heating around its edges. The second long range wake mitigation technique, referred to as detuning, consists in adjusting the frequencies of the deflecting dipole modes so as to make contributions from each cell interfere destructively.

The NLC Rounded Damped Detuned Structure (RDDS), has been described in numerous publications; it relies almost exclusively on detuning for long range wake mitigation. A very small amount of damping is introduced to suppress the recoherence that would occur periodically because of the necessarily finite number of cells in a structure. To that end, dipole energy is coupled out of each cell via four symmetrically arranged slots and channeled into longitudinal vacuum manifolds that also cylindrical waveguides. Each manifold is terminated by wideband matched absorber. The RDDS simultaneously achieves very effective long range wake suppression and maximum acceleration efficiency. However, this comes at the cost of challenging mechanical cell fabrication tolerances $(\sim 2-3 \mu$ on the cell dimensions or $\sim 2.5 \mathrm{MHz}$ on the dipole mode frequency) and alignment tolerances ( $\sim 15 \mu$ m cell-to-cell alignement). While it appears technically feasible to meet these tolerances, structure fabrication would be simplified if they could be relaxed without having to resort to some form of a posteriori control of the global error distributions (e.g. cell sorting).

An interesting avenue to explore is the possibility of relaxing tolerances in exchange for a small loss in efficiency. Preliminary calculations indicate that it should be possible to introduce enough cell-level damping to reduce the effective single cell dipole mode quality factor $Q$ from approximately a few 1000 down to about 500 without encountering serious localized overheating. It appears possible to limit the reduction in shunt impedance, compared with the standard RDDS cell, to $5 \%-10 \%$. 


\section{THEORY}

Consider a structure with $N$ cells and let the structure dipole modes frequencies denoted by $\omega_{p}$. Then, in absence of any damping mechanism, the net beam impedance experienced by a point charge traveling in a straight line along the structure is purely imaginary and given by

$$
S(\omega)=4\left\{Z_{\perp}(\omega)\right\}=4 \pi \sum_{p=1}^{N} K_{p} \delta\left(\omega-\omega_{p}\right)
$$

where $K_{p}$ is the kick factor associated with mode $p$. The function $S$, referred to in the literature as the spectral function, is a convenient quantity related to the impedance. Strictly speaking, $S(\omega)$ is the sine transform of the wake and, in constrast to the impedance, is defined for positive frequencies only. A useful result is that the wake envelope is the magnitude of the Fourier transform of $S(\omega)$. Assuming that the structure modes are localized in the vicinity of individual cells, equation (1) has a simple interpretation; it is merely the sum of the modal response of individual cells, as seen by a particle located as a distance $z$ behind a unit leading charge. In the case where the number of cells is sufficiently large, $S$ may be described by a smooth function $S(\omega) \simeq K \frac{d n}{d \omega} d \omega$, where $d n / d \omega$ is the spectral mode density (finite for $\omega>0$ and zero otherwise) and $K(\omega)$ is slowly varying function of the frequency. One then has, for the wake envelope

$$
e(z)=\left\|\int_{0}^{\infty} K(\omega) \frac{d n}{d \omega} \mathrm{e}^{j \omega z / c} d \omega\right\|
$$

One can see immediately that in order to reduce the extent of the wake envelope, one needs to increase the spectral function width. Furthermore, the product of the spatial extent and the spectral width is minimum when both distributions are Gaussian. This constitutes the basis of the RDDS design. In practice, the mode density distribution can only approximate the ideal Gaussian and deviates from it in two fundamental ways. Firstly, the distribution is truncated. The effect of this truncation can be understood easily by resorting to the convolution theorem. The wake envelope associated with a truncated Gaussian can be expressed as the convolution of product a Gaussian and the Fourier transform of a rectangle function. This transform is the well-known sinc function and accounts for the envelope oscillations observed after the initial decay of the envelope. For a given bunch separation, it is possible to tailor the truncated modal density so as to time the first zero of this wake envelope oscillation to roughly coincide with the passage of the next bunch. The second and more important deviation from the ideal Gaussian is associated with the discrete nature of the modal density. It leads to a natural division of the wakefield into two regions: a short range region for which the wake can be described by the average properties of the kick factors and mode density and a long range region $(z>c / \delta f)$ where $\delta f$ is a typical mode spacing. In the long range region, equation (2) no longer applies and it is necessary to resort to a numerical approach.

\section{NUMERICAL RESULTS}

To investigate the effect of damping on construction tolerances for damped detuned structures, an interactive program was developed. The program computes the wakefield by the spectral function method i.e. by solving deterministic equations in the frequency domain. Efficiency is maximized by making maximum use of the sparsity. Various construction errors can be modeled including individual cell dipole mode frequency errors, cell-to-cell transverse displacement errors termination errors (e.g. reflections) and cell-to-cell $Q$ variations (the absorber property). Figure 1 summarizes the effect of assembly errors on two structures: a $1.8 \mathrm{~m} 206$ cell RDDS and a fictitious MDS with identical length, number of cells and modal density distribution. It is assumed that the MDS would be using modified coupling slots and absorbers to produce localized damping equivalent to a cell $Q \simeq 500$. The $1.8 \mathrm{~m}$ structure was used because of readily available measurement and simulation data. However, although a 206 cell $1.8 \mathrm{~m}$ RDDS prototype was built and measured at SLAC, the NLC design is based on 100-cell $0.9 \mathrm{~m}$ structures. For the sake of reference, the long range wake effects in the NLC are negligible with respect to other sources of emittance dilution when the wake amplitude is roughly $<0.2 \mathrm{~V} / \mathrm{pC} / \mathrm{mm} / \mathrm{m}$.

On the first row are shown, plotted in red, the spectral function and wake envelope for an RDS, assuming a $\sigma=$ $100 \mu \mathrm{m}$ Gaussian error in cell-to-cell transverse alignment. For comparison, the corresponding error-free spectral function and envelope are also shown and plotted in blue. On the second row, results are presented for an MDS structure subjected to the same alignment errors.

The third and fourth rows of Figure 1 respectively show the spectral function and wake envelope for an RDDS and MDS subjected to a Gaussian dipole mode frequency error with $\sigma=1.0 \mathrm{MHz}$.

While the introduction of localized damping reduces the sensitivity to construction errors, its effectiveness appears to be significantly better in counteracting the effect of cell frequency errors (cell machining errors) than cell-to-cell transverse alignment errors. In particular, it can be seen that for distances $z<2 \mathrm{~m}$ the presence of localized damping has very little effect on the increased wake amplitude induced by cell alignment errors. Detailed calculations also show that the MDS equivalent cell $Q$ needs to be on the order of 500 or less to have a meaningful impact on tolerances. More $R \& D$ is required to firmly establish whether or not it is possible to obtain $Q<500$ while keeping localized losses under control.

\section{REFERENCES}

[1] K. Bane and R.L. Gluckstern, Part. Accel., 42, p123 (1993)

[2] N. Kroll et al. Manifold Damping of the NLC Detuned Accelerating Structure, SLAC-PUB-6660, 1994

[3] J.-F. Ostiguy, K.-Y. Ng, "Optimized wake field computations using a network model”, Linac 2000, Monterey CA, 2000 

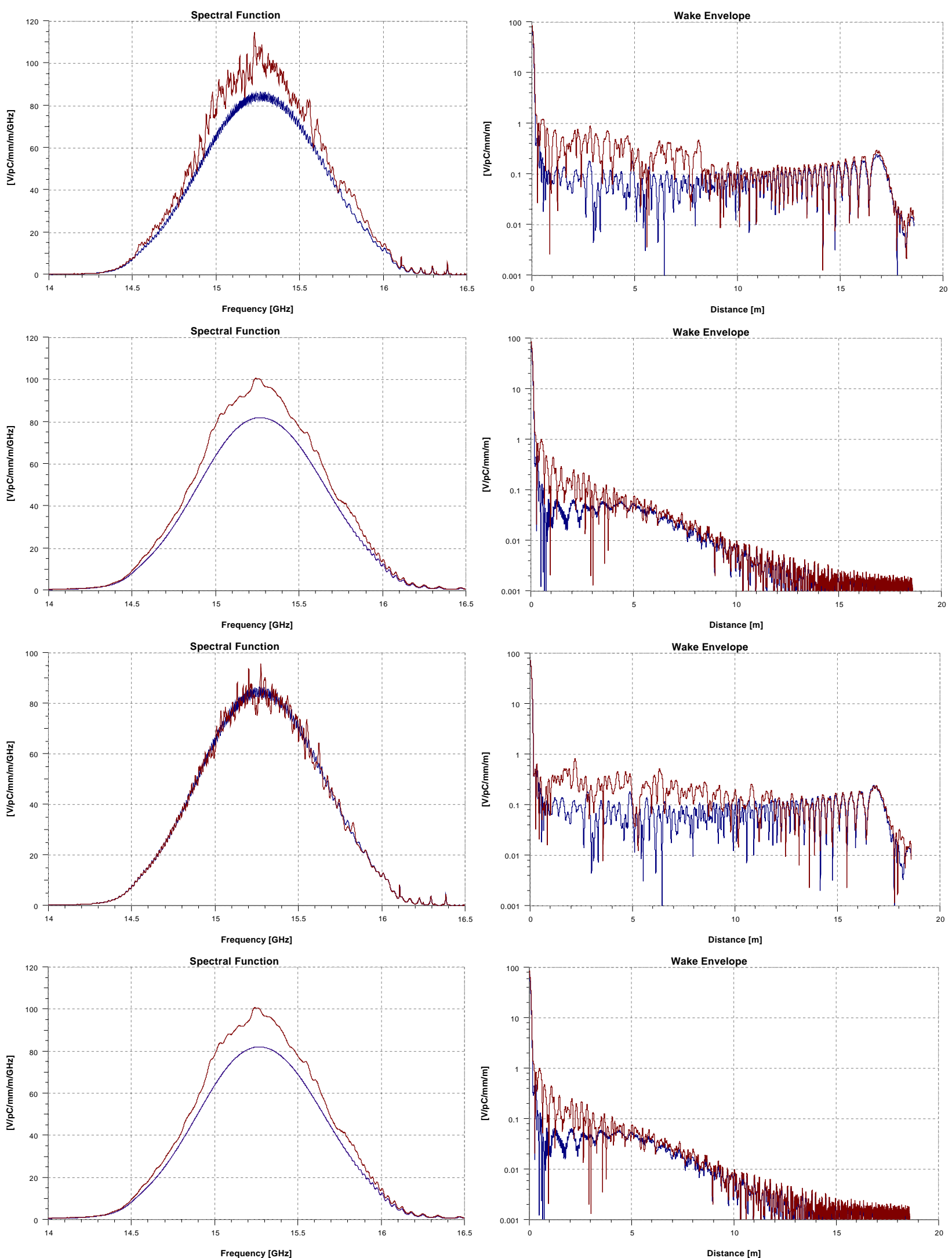

Figure 1: Spectral function and wake envelope for an RDDS and a MDS structure in presence of assembly errors. See text for explanation. 